\title{
Imaging the hypoglossal nerve
}

\author{
Pedro Alves* \\ Department of Radiology, Centro Hospitalar de Lisboa Central-Hospital de São José, Rua José António Serrano, 1150-199 Lisboa Codex, Portugal
}

\section{A R T I C L E I N F O}

\section{Article history:}

Received 24 August 2009

Accepted 27 August 2009

\section{Keywords:}

Hypoglossal nerve

Hypoglossal nerve pathology

Magnetic resonance imaging

CT

\begin{abstract}
A B S T R A C T
The hypoglossal nerve is a pure motor nerve. It provides motor control to the intrinsic and extrinsic tongue muscles thus being essential for normal tongue movement and coordination. In order to design a useful imaging approach and a working differential diagnosis in cases of hypoglossal nerve damage one has to have a good knowledge of the normal anatomy of the nerve trunk and its main branches. $A$ successful imaging evaluation to hypoglossal diseases always requires high resolution studies due to the small size of the structures being studied. MRI is the preferred modality to directly visualize the nerve, while CT is superior in displaying the bony anatomy of the neurovascular foramina of the skull base. Also, while CT is only able to detect nerve pathology by indirect signs, such as bony expansion of the hypoglossal canal, MRI is able to visualize directly the causative pathological process as in the case of small tumors, or infectious/inflammatory processes affecting the nerve. The easiest way to approach the study of the hypoglossal nerve is to divide it in its main segments: intra-axial, cisternal, skull base and extracranial segment, tailoring the imaging technique to each anatomical area while bearing in mind the main disease entities affecting each segment.
\end{abstract}

(C) 2010 Elsevier Ireland Ltd. All rights reserved.

\section{Introduction}

The twelfth cranial nerve or hypoglossal nerve is an entirely motor cranial nerve that controls the intrinsic and extrinsic (genyoglossus, hyoglossus and styloglossus) muscles of the tongue and also the infrahyoid strap muscles through the ansa cervicalis [1]. Although uncommon, damage to the hypoglossal nerve produces characteristic clinical and radiological manifestations of which unilateral tongue atrophy is the most common [2-4].

Various pathological entities may affect the nerve, and, like the other cranial nerves, it is common to divide them in supranuclear, nuclear and infranuclear causes.

However, for the radiologist approaching a patient with hypoglossal nerve damage it is especially useful, in addition to the above mentioned classification, to bear in mind the various anatomical segments of the nerve and organize the diagnostic work-up according to the segment(s) that present pathological changes.

The hypoglossal nerve can be divided in roughly four segments: intra-axial, cisternal, skull base and extracranial segment which can be damaged by different pathological processes. Knowledge of the different disease entities affecting each segment narrows the differential diagnosis and allows a more focused diagnostic evaluation.

\footnotetext{
* Tel.: +35121 884 1000; fax: +35121884 1023

E-mail address: tojais.pedro@gmail.com.
}

This article reviews the normal anatomy of the hypoglossal nerve highlighting the most important features from a radiological perspective, presents the available imaging techniques to study the nerve and reviews the most common pathological conditions affecting the various anatomical segments.

\section{Anatomy}

\subsection{Medullary segment}

The hypoglossal nucleus is located in the dorsal medulla between the midline and the dorsal vagal nucleus. It is an elongated thin nucleus that extends in a caudal-cranial direction along the medulla oblongata for about $18 \mathrm{~mm}$ [5-9]. In its most inferior portion it causes a bulging of the floor of the fourth ventricle called the hypoglossal eminence or trigone (Fig. 1).

The axons that will form the hypoglossal nerve course in a ventral direction through the medulla, internal to the medial lemniscus. The fibers exit at the ventrolateral sulcus or pre-olivary sulcus between the olivary nucleus and the pyramid by way of several thin rootlets in an area designated by root exit zone.

The hypoglossal nucleus has connections with fibers from the glossopharyngeal, vagus and trigeminal nerves, mediating reflex movements in response to a wide variety of stimuli from the lingual, oral and pharyngeal mucosa, that are important for the processes of speaking and swallowing.

The nucleus receives its blood supply through the anterior spinal and vertebral arteries. 

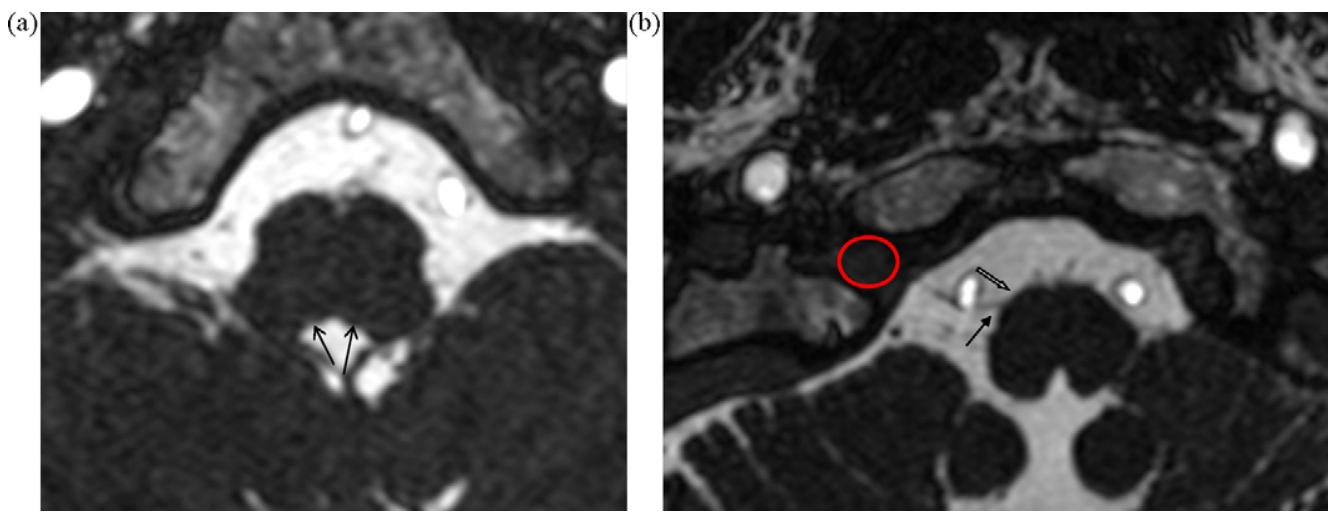

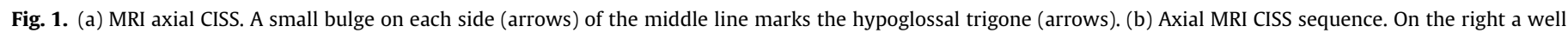
defined rootlet is seen (arrow). The pre-olivary sulcus is seen adjacent to it (open arrow). The right hypoglossal canal is well defined (circle).

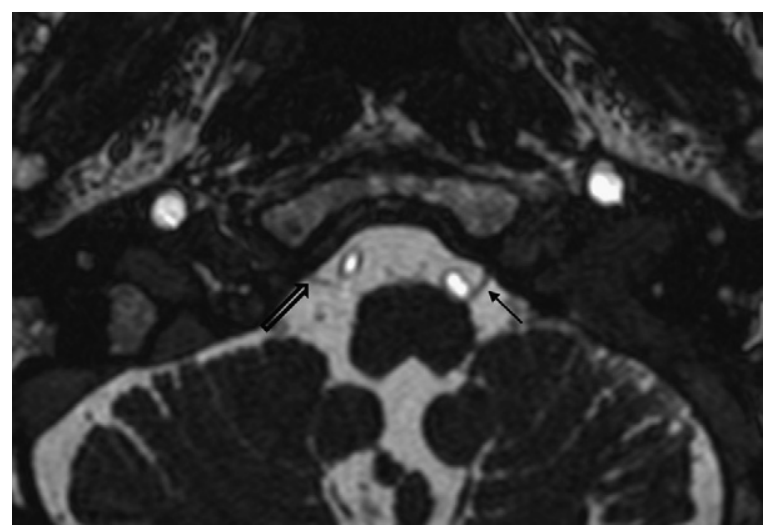

Fig. 2. Axial CISS. The left hypoglossal nerve (arrow) emerging from the medulla as a single trunk and entering the hypoglossal canal. A thinner rootlet is seen on the right (open arrow).

\subsection{Cisternal segment}

The pre-medullary cistern contains the rootlets of the nerve, formed by the coalescence of multiple efferent fibers. These rootlets are in close proximity with the vertebral artery, being located posterolateral to it. Just before exiting the skull base at the hypoglossal canal the rootlets fuse, forming the hypoglossal nerve (Figs. 2 and 3).

In the cisternal segment small nerve filaments may also fuse with some vagal nerve fibers.

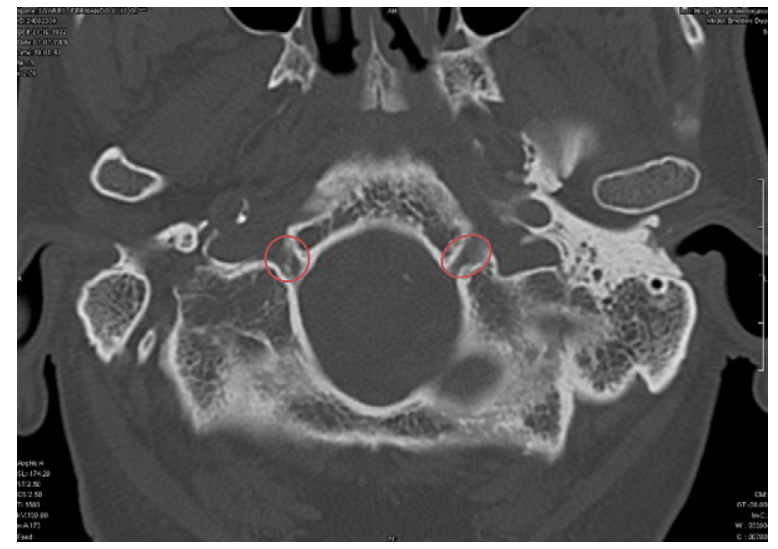

Fig. 3. Axial CT scan showing both hypoglossal canals with smooth well corticated margins (circles)

\subsection{Skull base segment}

This segment is formed by the nerve inside the hypoglossal canal, a structure within the occipital bone inferiorly to the jugular foramen (Figs. 4, 5 and 7).

The nerve is surrounded at this level by a venous plexus, a feature that allows its study by contrast enhanced T1WI MRI sequences, and also by a meningeal branch of the ascending pharyngeal artery. Identification of these vessels, especially the venous plexus, may be important because an enlarged emissary vein draining into the sigmoid sinus may be responsible for some cases of hypoglossal palsy $[10,11]$.

The canal pierces the occipital condyle in a lateral and forward direction and it may be divided by a small bony spicule.

\subsection{Extracranial segment-carotid space segment}

The hypoglossal nerve exits the canal and gives origin to some small dural branches just before entering the nasopharyngeal carotid space at its medial side. It then descends in close contact with the vagus nerve and exits the carotid space between the internal jugular vein and the carotid artery taking an anterior course and lying at the inferior border of the posterior belly of the digastric muscle.

\subsection{Extracranial segment-anterior segment}

After exiting the carotid space the nerve continues anteriorly and inferiorly towards the hyoid bone and at the level of the occipital artery base passes below the posterior belly of the digastric muscle.

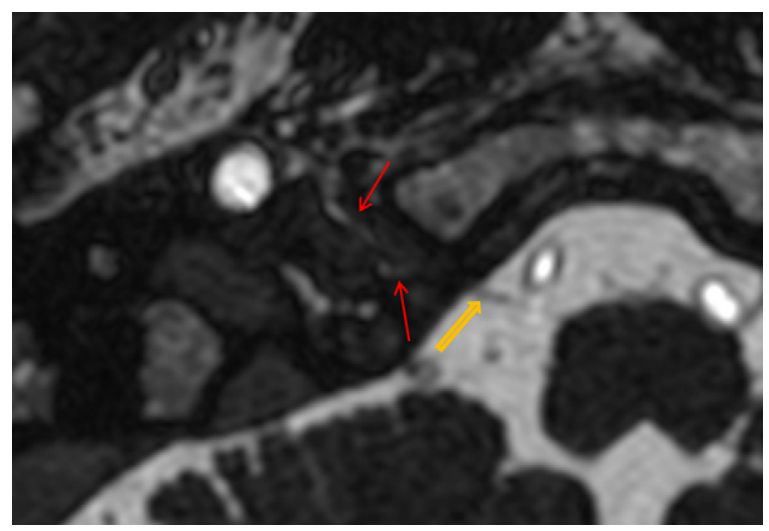

Fig. 4. Axial CISS: normal vascular structures inside the hypoglossal canal (arrows). A thin rootlet of the right hypoglossal nerve is seen (yellow arrow). 

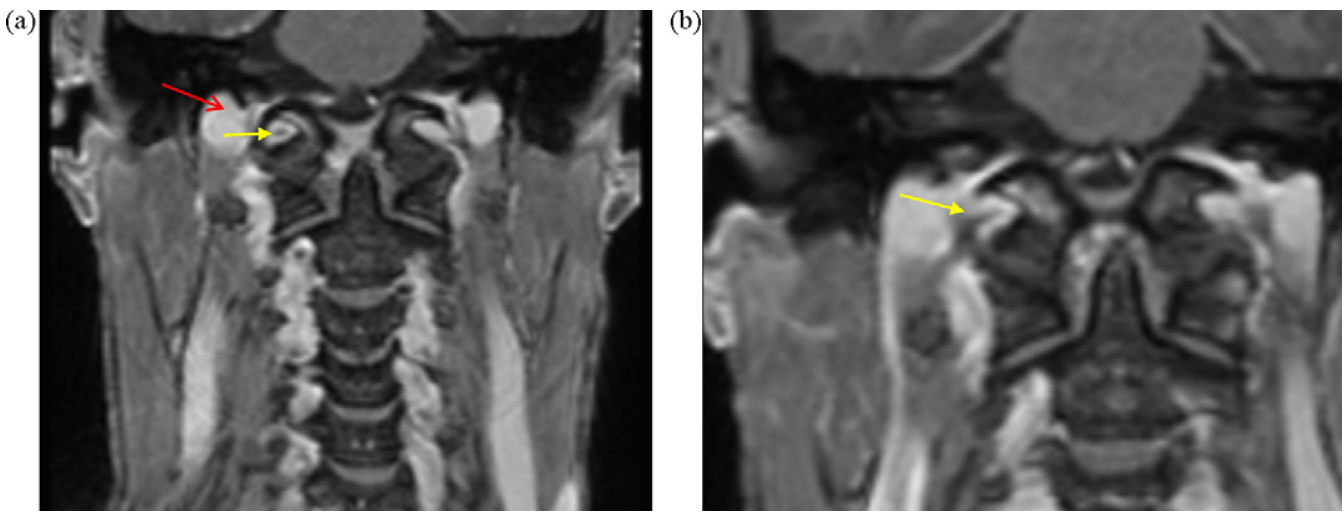

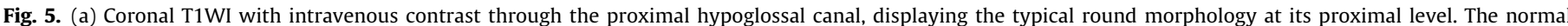

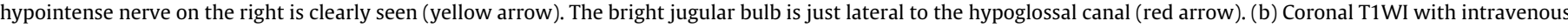

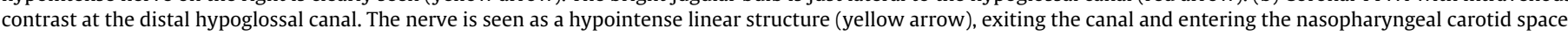

The terminal segment of the nerve gives off its distal branches, the most important being the muscular branch on the lateral border of the hyoglossus in the posterior sublingual space (Fig. 6) and the ansa cervicalis that together with the superior and inferior spinal roots of $\mathrm{C} 1-\mathrm{C} 3$ innervates the infrahyoid strap muscles.

\section{Imaging strategy}

The need for imaging in cases of hypoglossal nerve palsy arises from the limitations of the clinical examination. By itself, the clinical examination cannot localize with accuracy the site of damage to the nerve. The best one can accomplish, based on the inspection of the tongue and analysis of the function of the other cranial nerves is to differentiate between supranuclear, nuclear and infranuclear pathology.

In most of the clinical scenarios, albeit some minor differences, damage to the hypoglossal nerve produces the same tongue changes regardless of the segment being affected, and imaging is essential to make an accurate topographical diagnosis as well as to define its cause.

Regardless of the imaging technique that the radiologist chooses there are two basic principles that must be adhered to.

The first is that the entire anatomical course of the nerve should always be covered. This is essential in order to have a full picture of the different segments of the nerve and to accurately map the

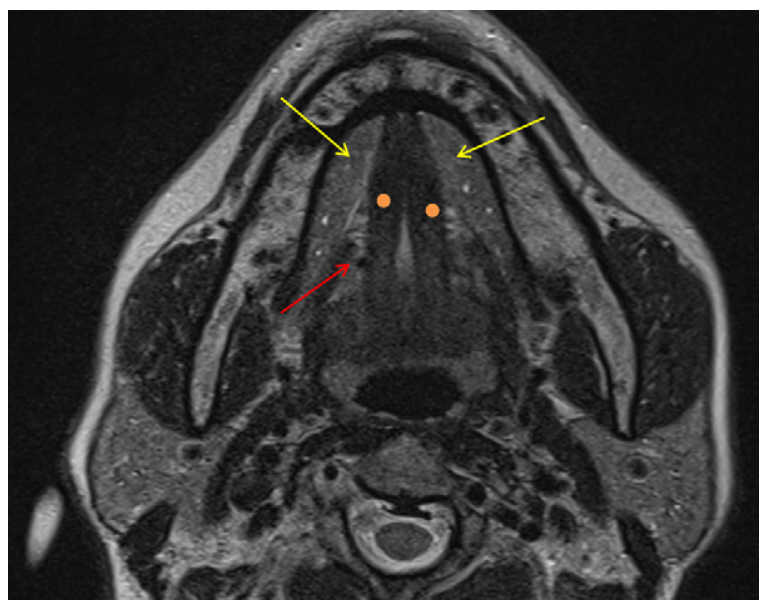

Fig. 6. Axial T2WI through the sublingual space. The hypoglossal nerve cannot be seen but its position can be inferred in the sublingual space adjacent to the vascular bundle. Just medial to the hyoglossus one can see the circular shape of the lingual artery (arrow). Sublingual glands (yellow arrows), Genioglossus (orange circle). offending lesion, but it is especially important when one is staging a head and neck malignancy, where perineural spread of the primary tumor with possible multiple skip lesions may occur along the nerve course, even without any clinical signs.

The second is the obligatory use of intravenous contrast. With MRI, gadolinium enhanced T1WI sequences are very often the only ones that allow the diagnosis of malignant perineural spread, infection or inflammation of the nerve and of small tumors. On CT, although the nerve proper is not visualized, the use of contrast, especially in cases of tumors, increases lesion conspicuity and determines the important vascular relationships.

Conventional radiology and ultrasound are insufficient to give useful information concerning the hypoglossal nerve and today the diagnostic approach to nerve dysfunction, as in the case of other cranial nerves, relies on CT and MRI. CT and MRI have a complimentary role. CT displays the osseous anatomy with exquisite detail while MRI has superior soft tissue contrast. Both allow the study of the perineural fat pads, the loss of which signals pathological nerve involvement.

The new generation of volumetric CT and high field MR scanners allows higher resolution and faster imaging studies, depicting the various nerve branches and mapping the entire cranial nerve course.

\section{1. $C T$}

Although MRI is superior in visualizing the nerve proper, its rootlets and branches, CT easily displays the dimensions and morphology of the hypoglossal canal, and the state of the neighboring foramina and osseous segments of the skull base, information that is especially relevant to the pre-operative planning.

From a radiological perspective, the bone changes displayed by the pathological entity under study allow one to make the differential diagnosis between a rapidly destructive process that creates a moth eaten or permeative appearance or one with a more slow or indolent course that tends to remodel and expand the bone segments in the vicinity [12].

As stated above, the CT protocol for study of the hypoglossal nerve must include the entire course of the nerve from its origin to the inferior distal branches, which means scanning from the orbital roof down to below the hyoid bone. Newer scanners easily acquire $1 \mathrm{~mm}$ or thinner slice thickness allowing isotropic or near-isotropic reconstructions in any plane desired. Regardless of the scanner used, in order to obtain a diagnostically useful study of the skull base and its bony foramina, the slice thickness should not exceed $3 \mathrm{~mm}$ (Table 1 ). 
Table 1

CT parameters for study of the hypoglossal nerve/skull base foramina.

\begin{tabular}{|c|c|c|c|c|}
\hline Parameters & Single slice & $4-8$ slices & 16 slices & 64 slices \\
\hline Collimation (mm) & 1 & $1 / 1.25$ & $1.25 / 1.5$ & $0.6 / 0.625$ \\
\hline Reconstruction increment & $1 \mathrm{~mm}$ & $3 \mathrm{~mm}$ (1 mm-recon) & $2-3 \mathrm{~mm}$ (1 mm-recon) & $2-3$ mm (0.6 mm-recon) \\
\hline Slice thickness & $3 \mathrm{~mm}$ & $3 \mathrm{~mm}$ (1.5 mm-recon) & $2-3 \mathrm{~mm}$ (1.5-2 mm-recon) & $2-3 \mathrm{~mm}$ (0.75-1 mm-recon) \\
\hline Convolution kernel & Bone/soft tissue & Bone/soft tissue & Bone/soft tissue & Bone/soft tissue \\
\hline Intravenous contrast & Yes & Yes & Yes & Yes \\
\hline Concentration (mg iodine/ml) & 300 & 300 & 300 & 300 \\
\hline Volume (ml) & $90-100$ & $90-100$ & $70-100$ & $70-100$ \\
\hline Injection rate $(\mathrm{ml} / \mathrm{s})$ & $2-3$ & $2-3$ & $2-3$ & $2-3$ \\
\hline Delay (s) & $50-70$ & $50-70$ & $50-70$ & $50-70$ \\
\hline
\end{tabular}

If there is the possibility of multiple dental artifacts and the scanner allows it (usually only possible with single slice or 4-slice scanners), two spirals should be acquired, angulated conversely to avoid the teeth. The first spiral that covers the skull base should be acquired with the thinnest collimation possible $(\leq 1 \mathrm{~mm})$ while the second spiral covering the rest of the neck may be acquired with a thicker collimation ( 2 and $5 \mathrm{~mm}$ ).

Contrast should be administered and if possible a CTangiographic study obtained. Contrast enhances lesion detection and characterization and allows the study of the relationship of the lesion and nerve with nearby vascular structures. The best results are achieved at a diagnostic window of about 50-70 s after the start of the injection, allowing for a good discrimination between the enhancing tumor rim and the surrounding soft tissues.

\section{2. $M R I$}

MRI provides exquisite soft tissue contrast and allows direct visualization of the different segments of the nerve, and generally it is the method of choice for the study of a patient presenting with hypoglossal nerve palsy of unknown cause. The main problem with MR studies is that they are time consuming and more prone to motion artifacts requiring a good collaboration from the patient.

A high field scanner of $1 \mathrm{~T}$ or higher is necessary to produce images that are diagnostically useful.

Multiple sequences are available to study the nerve and each has specific advantages according to the segments being studied. The principles of designing a MR protocol for the hypoglossal nerve follow the same basic principles as for the other cranial nerves [7,13-15,38] (Table 2).

For the intra-axial segment or above (nuclear or supra-nuclear lesions) a brain protocol is used with fast spin-echo (FSE) T2W images, diffusion weighted imaging DWI and T1W pre- and postcontrast.

For the cisternal segment a 3DFT heavily T2W sequence (CISS, DRIVE, FIESTA) with submillimiter thickness (usually $0.7 \mathrm{~mm}$ ) should be used. The nerve, its rootlets and filaments stand out as hypointense structures surrounded by hyperintense CSF.

For the evaluation of neurovascular conflicts the use of 3DFT TOF MRA (3D FISP or FLASH) permits an accurate assessment of the relationship of the vessels to the nerve or its components.

High resolution T1W images pre- and post-GAD with fat suppression (SPGR, GRASS, MPRAGE), allow further anatomical characterization of the remaining segments. The use of contrast is especially important in infectious/inflammatory pathology where abnormal enhancement of the nerve may be the only abnormality depicted.

CISS images are especially useful in the axial plane, demonstrating the take-off of the nerve rootlets in the cisternal segment.

The hypoglossal canal should be routinely studied in the axial and coronal planes. Sometimes the sagital plane may be useful, depending on the pathological changes.
The extracranial segment should be imaged in the axial and coronal planes. The sagital plane is also useful for distal lesions involving the floor of mouth or tongue base.

\section{Imaging landmarks for identifying the hypoglossal nerve}

Various approaches exist to film reading and each radiologist uses the one with which he or she is familiar. Most important is to have a systematic approach and to use easily and consistent identifiable anatomical landmarks.

In the intra-axial segment one should look for the bulging on the floor of the fourth ventricle that marks the site of the nucleus.

Then one should turn the attention to the pre-olivary sulcus that roughly locates the root exit zone for the proximal cisternal segment of the root bundles.

The hypoglossal canal is a more easily identifiable landmark. In axial images it is very easy to locate. One possible strategy is to first identify the atlanto-occipital joint, then the condyles and finally the canal as a bony defect with a tubular shape. In the coronal plane the proximal canal has a circular shape while the more distal parts having a "birds head and beak" appearance. On coronal images the nerve is located roughly in the lateral-superior quadrant of the canal. The sagital plane is slightly difficult to interpret. The easiest way to begin is to identify the atlanto-occipital joint by its superior concave surface and then immediately above it locate the enhancing venous plexus inside the hypoglossal canal and the hypointense round nerve (Fig. 7).

Direct visualization of the hypoglossal nerve in the carotid and sublingual space is more difficult in these segments and its position is inferred by the adjacent anatomical structures as described in the above section.

\subsection{Clinical signs of hypoglossal pathology}

Unlike other cranial nerves, lesions of the hypoglossal are rare and when present they are usually associated with other nerve palsies.

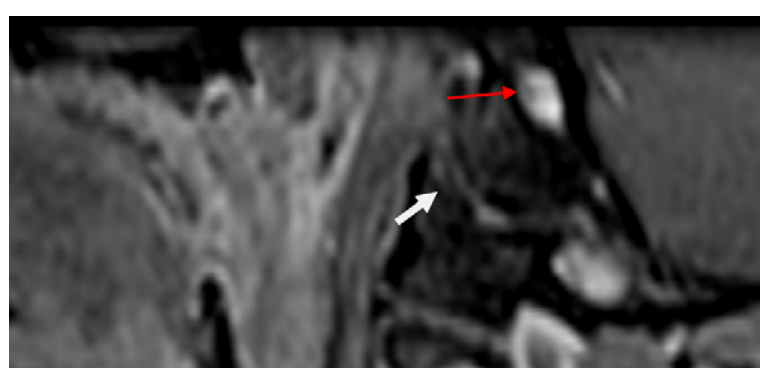

Fig. 7. Sagital T1WI with intravenous contrast: the curved upper surface of the atlanto-occipital joint (white arrow) and above it the round hypoglossal canal with the enhancing venous plexus and a small hypointense dot representing the nerve (thin black arrow). 
Table 2

MR sequences for the evaluation of cranial nerves (adapted from Borges [14]).

\begin{tabular}{|c|c|c|c|c|c|}
\hline Parameters & FSE T1Wa (GAD) & TSE T2W & 3DFT MPRAGE ${ }^{\mathrm{a}}$ (GAD) & 3DFT CISS ${ }^{\mathrm{a}}$ & 3DFT-FISP turbo-MRA ${ }^{a}$ \\
\hline TR & 684 & 4000 & 11.6 & 12.25 & 35 \\
\hline $\mathrm{TE}$ & 20 & 99 & 4.9 & 5.9 & 6.4 \\
\hline FA & $90^{\circ}$ & $180^{\circ}$ & $12^{\circ}$ & $70^{\circ}$ & $15^{\circ}$ \\
\hline Thickness & $2 \mathrm{~mm}$ & $4 \mathrm{~mm}$ & $1 \mathrm{~mm}$ & $0.7 \mathrm{~mm}$ & $0.75 \mathrm{~mm}$ \\
\hline Matrix & $160 \times 256$ & $242 \times 512$ & $192 \times 256$ & $192 \times 256$ & $320 \times 512$ \\
\hline FOV & 230 & 300 & 240 & 95 & 200 \\
\hline
\end{tabular}

a Other similar sequences that may be used include: FFE T1W, fast field echo T1W; 2D FLASH GE, bi-dimensional fast low-angle shot gradient echo T1WI; 3D-GRASS,

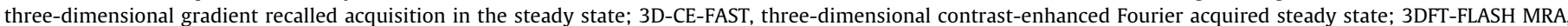

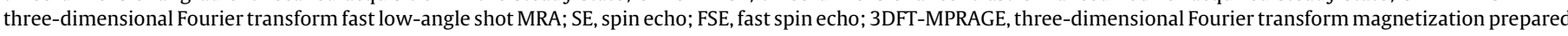

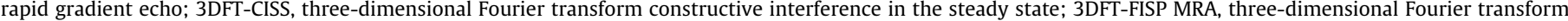
fast-in-flow steady-state precession 2115.

The multiple causes [18-20] of hypoglossal palsy may be divided according to their etiological type (Table 3 ).

Malignant tumors are the most common cause of hypoglossal nerve palsy (HNP). Other causes are trauma, infection, medullary infarction, multiple sclerosis and Guillain Barré Syndrome [16].

Isolated HNP is an even rarer condition and when present usually poses a diagnostic challenge with frequent misdiagnosis.

The hypoglossal nerve is a pure motor nerve and nerve damage leads to imbalance of the tongue muscles causing deviation towards the weak side.

When the lesion is supranuclear there is weakening/paralisys of the tongue on the contralateral side of the lesion but this is usually compensated by the normal side and so, tongue weakness in these cases tends to be mild.

When the lesion is nuclear or infranuclear the clinical changes are ipsilateral and the tongue deviates towards the side of the lesion, associated with muscle atrophy and fasciculation, signs that point towards a lower motor neuron disease.

Nuclear lesions are often associated with other signs of brainstem involvement, with combined neurologic deficits.

If the clinical examination also shows a disorder of the infrahyoid strap muscles (Fig. 8) the physician can localize the lesion to one of the segments distal to the point where the hypoglossal nerve joins the $\mathrm{C} 1$ fibers - the carotid and anterior segments.

\subsection{Pathology}

In the majority of cases the first radiological sign that draws attention to a lesion of the nerve is unilateral atrophy of the tongue.

The imaging approach consists of analyzing the different segments of the nerve and recognizing the most common differential diagnosis [21] in each (Table 4). It is not possible to provide an extensive review of all the lesions that can cause hypoglossal nerve palsy and so the discussion will be limited to the most common entities.

\subsubsection{Tongue}

The first step is to make sure that true tongue atrophy is present. Lesions with a prominent fatty component in the sublingual space such as lipomas or dermoids that are in close contact and displace the tongue musculature may be confused with an intrinsic lesion of the tongue and should be systematically excluded.

If a true atrophy is present then there will be volume loss on the affected side with fat density on CT (Fig. 8) and fat intensity on the MR sequences.

Imaging changes in the tongue after damage to the hypoglossal nerve vary according to the time elapsed between the initial nerve insult and the imaging examination [2,22-25].

After the hypoglossal nerve has been damaged the axons downstream to the site of the lesion degenerate and the muscles dependent on the nerves affected begin to denervate. Muscle volume begins to decrease secondary to atrophy of the muscle fibers. As more time passes fibrous changes and fatty infiltration ensue.

The most useful imaging modality to characterize the various muscle changes is MRI. In the subacute phase of denervation the tongue is hyperintense on T2WI and hypointense on T1WI, mainly due to the increased water content of the interstitial space. If intravenous contrast is given there may be an increased uptake due to the relative increase in capillaries per unit volume in the atrophic muscle and the accumulation of contrast material in the increased interstitial compartment. In this initial phase the CT appearance is nonspecific.

In the chronic phase as fatty infiltration progresses, the tongue volume on the affected side will decrease and on axial images one will see posterior protrusion of the base of the tongue. On MRI the affected tongue will have high signal on both T1WI and T2WI and on CT the characteristic fat density will be noted. On T1WI, areas of hyperintensity will begin to appear at about five months after the initial insult.

The radiologist should be alert to the various appearances of tongue denervation. It is especially important, when there is loss of volume on one side, not to confuse the unaffected side with a mass and avoid biopsying the normal tongue in search of a tumor.

Table 3

Causes of hypoglossal nerve palsy.

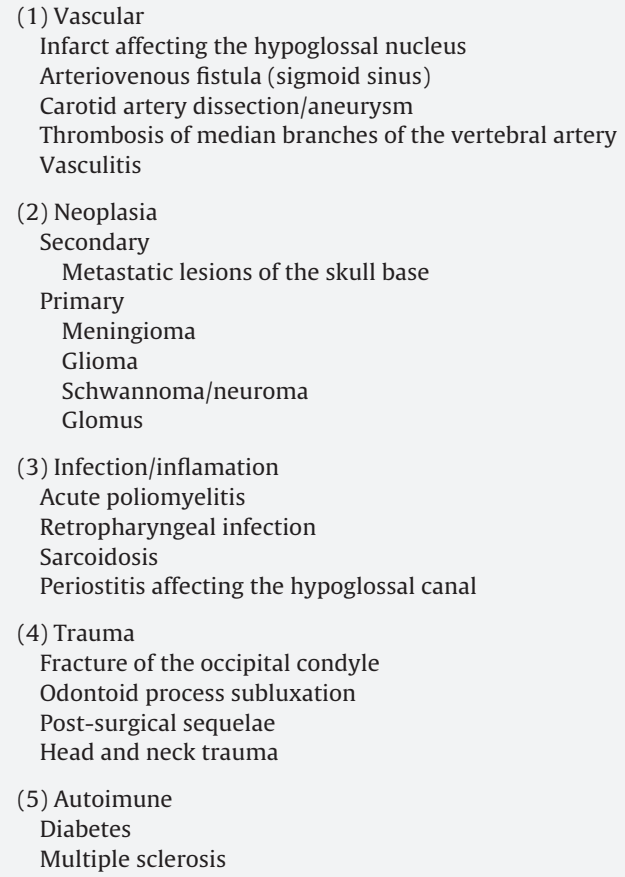



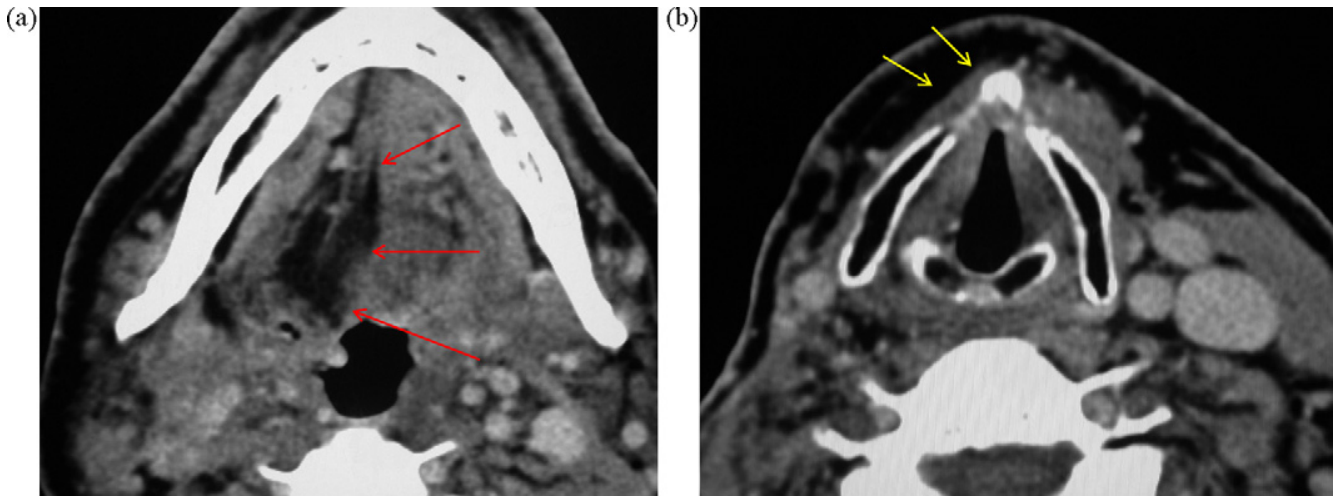

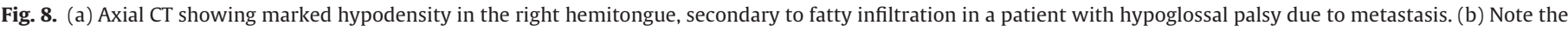
decreased thickness of the pre-laryngeal strap muscles, signaling involvement of the ansa cervicalis.

\subsection{Medullary segment}

A variety of disease processes may affect the hypoglossal nucleus. Pathology of this segment of the nerve is frequently associated with damage to other cranial nuclei resulting in a complex lower cranial neuropathy [19]. If both nuclei are involved the result is complete paralysis of the tongue.

Neoplasms are among the most common causes and may be primary or secondary $[16,17]$. Most frequent are metastases followed by gliomas. Metastasis can have a variety of imaging appearances and should always be high on the differential diagnosis of space-occupying brainstem lesions. Gliomas are heterogeneous infiltrative lesions featuring high signal intensity on T2WI and FLAIR sequences and causing expansion of the brainstem in a patient presenting with multiple cranial nerve deficits.

Other less common disease processes of inflammatory, infectious or demyelinating nature may also occur, such as multiple sclerosis, amyotrophic lateral sclerosis or poliomyelitis [26]. The best technique to study these lesions is MRI. In the case of multiple sclerosis, typical high signal intensity plaques along the nucleus are seen in PD, T2WI and FLAIR sequences.

Infectious causes of isolated hypoglossal palsy are extremely rare, with only a few cases described in the literature, mainly in

Table 4

Segmental differential diagnosis of hypoglossal palsy.

(1) Medullary segment

Infartcion (ischemia/hemorrhage)

Neoplasia (glioma)

Multiple sclerosis/demyelinating diseases

(2) Cisternal segment

Neurovascular conflict (PICA, VA)

Aneurysm

Vascular ectasia

Neoplasia (meningioma)

Rheumatoid arthritis

(3) Skull base segment

Skull base metastasis

Nasopharyngeal carcinoma

Nerve sheath tumor

Glomus tumor

(4) Extracranial segment-carotid space Carotid artery dissection/aneurysm Neoplasia (squamous cell carcinoma) Adenopathy (metastatic/inflammatory) Vascular intervention

(5) Extracranial segment-anterior segment Infection/inflammation - sublingual space Squamous cell carcinoma

Post-surgical sequelae children, and its features are not well recognized due to the small number of cases reported.

\subsection{Cisternal segment}

The cisternal segment lies in the pre-medullary cistern and it may be affected by intrinsic or extrinsic pathological processes to the nerve.

Extrinsic lesions are usually the result of direct extension of skull base pathology, usually neoplasms (chordoma and meningioma), but also some infectious/inflammatory causes such as skull base osteomyelitis.

Primary neoplasms of the hypoglossal nerve may also occur but are uncommon [27-31]. As with other neurinomas elsewhere they are classified in three types, according to their location along the nerve. In decreasing order of frequency they are type B (intracranial and extracranial 50\%), type A (intra-cranial 31.5\%) and type C (peripheral 18.5\%). Type B has a characteristic dumbbell shape.

Patients with a hypoglossal neurinoma (Fig. 9) may present in several distinct ways, depending on the location of the tumor. Type B lesions usually present as a combined palsy of the nerve (due to the intra-canalicular component) with more or less extensive involvement of the other cranial nerves. Type $\mathrm{C}$ lesions present as a neck mass, in the carotid or submandibular space, and the palsy will depend on the number of nerve fibers involved. Type A neurinoma may produce multiple cranial nerve deficits, but interestingly, the cases of hypoglossal neurinomas without any clinical signs of palsy all had their origin in the intra-cranial nerve rootlets, CT will show regular expansion of the hypoglossal canal by the neurinoma, with bone remodeling but without rupture of the cortex. The tumor itself is slightly hypodense and enhances after contrast. It usually has an oblong shape, along the nerve course. With bigger lesions one finds increasing structural heterogeneity with cystic areas, hemorrhage or necrosis. On MRI neurinomas are usually hypointense on T1WI, hyperintense on T2WI and also show strong enhancement.

Vascular abnormalities such as an aneurysm of the vertebral artery or dolichoectasia may cause compression of the nerve and subsequent clinical symptoms. However, anatomical and radiological studies show that both the VA and the PICA may deform, compress or displace the twelfth nerve regardless of the patient's clinical symptoms, making the visualization of these contacts of doubtful pathological significance in an entirely asymptomatic patient.

Although not as well studied as for other cranial nerves, determining what constitutes a true neurovascular conflict on imaging is subject to controversy with some authors advocating the use of very specific and strict criteria to make this diagnosis [32]. Some of 

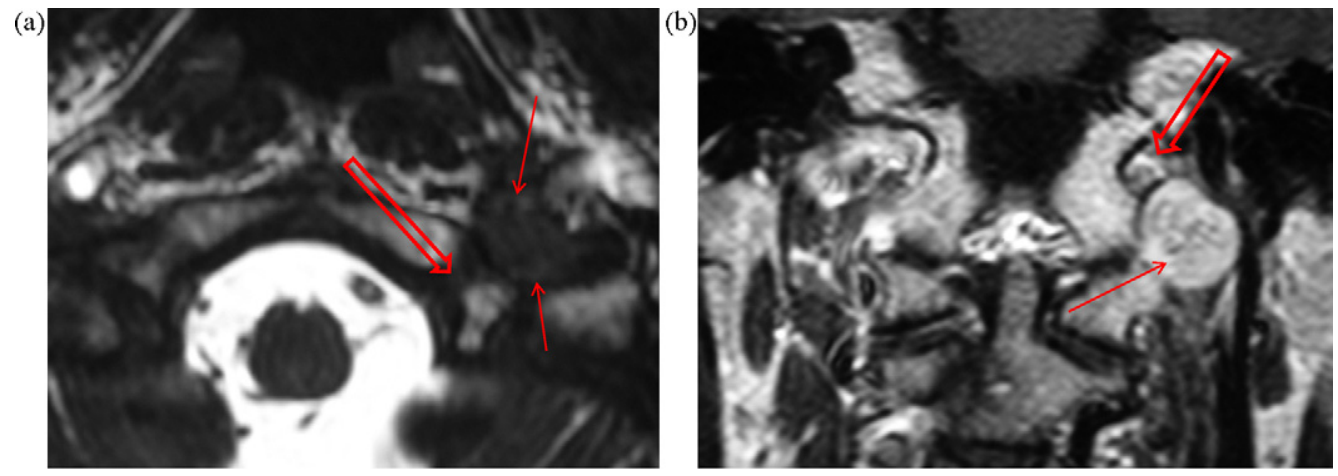

(c)

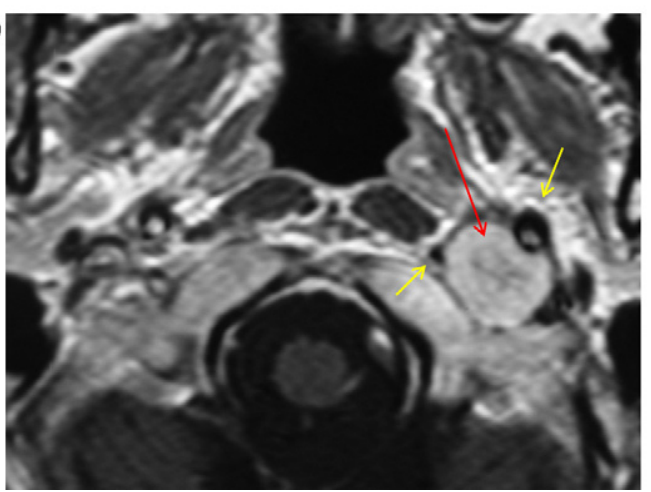

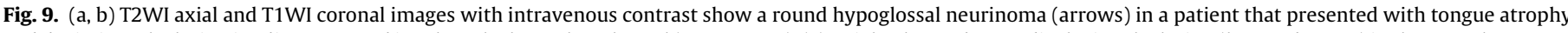

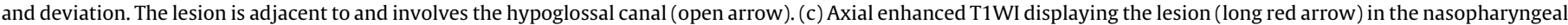
carotid space splaying the vessels (short yellow arrows).

these criteria are that the location of the conflict should be at the root entry zone, the vessel must cross the nerve perpendicularly, the nerve must show some displacement and the vessel in question must be an artery (Figs. 10 and 11).

As in the case of other nerves, the best MRI sequence to study neurovascular conflicts is the CISS sequence. MRA sequences are less sensitive if the vascular structures involved are veins, due to the slow blood flow inside these vessels.

Infection at the skull base such as osteomyelitis [33] or subarachnoid hemorrhage may also cause damage to the nerve, its rootlets and filaments.

Alterations in the neighboring osseous segments of the skull base by trauma or congenital alterations are also potential sources of nerve damage. Abnormal locations of the medulla in the foramen magnum, such as in Arnold-Chiari malformation or odontoid

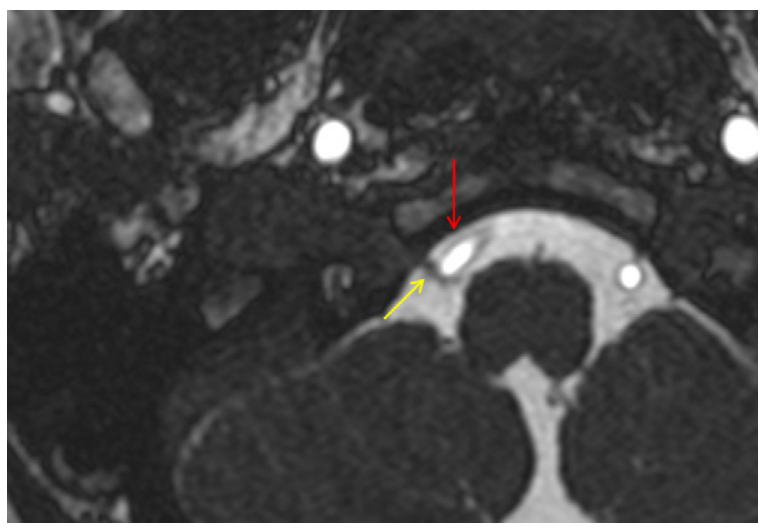

Fig. 10. Axial CISS. Slightly thickened right hypoglossal nerve (yellow arrow) in close contact with the ipsilateral vertebral artery (red arrow). The patient had no symptoms of hypoglossal nerve palsy. impingement in rheumatoid arthritis, are examples of conditions that may be associated with HNP.

\subsection{Skull base segment}

A variety of disease processes [34] may involve the hypoglossal nerve at this level with the most common causes of nerve damage being tumors and trauma.

Both benign and malignant tumors may damage the nerve. They extend from nearby segments of the skull base and cause erosion or expansion of the nerve canal. The most common malignant tumors are metastasis from breast, lung and prostate cancer and direct extension of nasopharyngeal carcinoma through the skull base (Figs. 12 and 13).

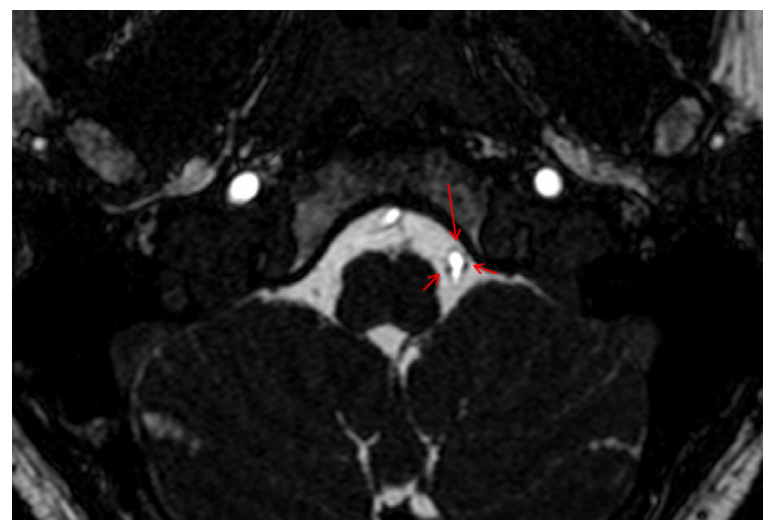

Fig. 11. Axial CISS. The left hypoglossal nerve forming a small "loop" that is crossed by the ipsilateral vertebral artery (long arrow), seen as two hypointense dots (short arrows) around the vessel. 


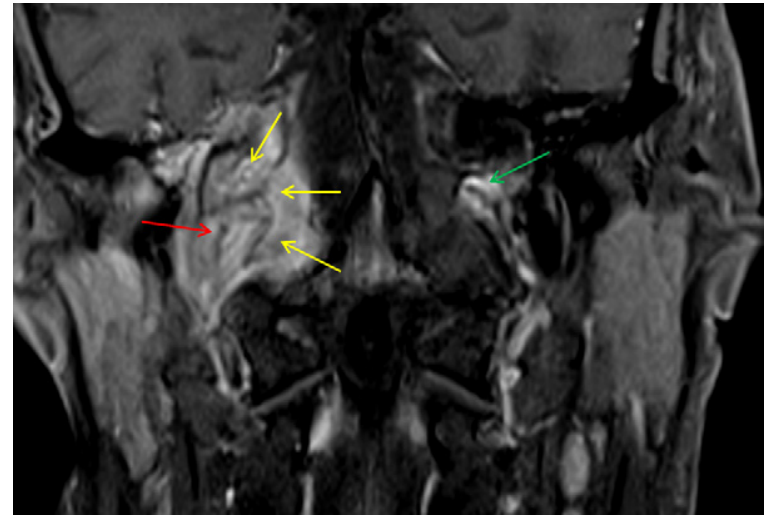

Fig. 12. Coronal T1WI with intravenous contrast. Extensive metastatic involvement of the right hypoglossal (yellow arrows) canal and nerve (red arrow) with marked post-contrast enhancement of the osseous segments and the interior of the canal. The left hypoglossal canal and nerve are normal (blue arrow).

Bone tumors may also affect the nerve, secondary to the osseous changes that ensue. The differential diagnosis is extensive and includes giant cell tumors, osteogenic sarcomas and cartilaginous lesions. In the same manner primary bone lesions such as fibrous dysplasia or Paget's disease that involve the base of the skull also cause nerve dysfunction due to compression of various bone foramina.

Benign tumors include meningiomas, glomus tumors and peripheral nerve sheath tumors [27].

Infections of the skull base [33], especially in diabetic or immunocompromised patients are another cause of nerve dysfunction and should be included in the differential diagnosis. Among these are Pseudomonas and Tuberculous osteomyelitis.

\subsection{Extracranial segment}

Although the differential diagnosis of lesions affecting the extracranial segment of the nerve is extensive, in daily practice most of the cases are due to malignant tumors [16], either by direct invasion by the primary mass or by perineural spread along the nerve trunks.

Perineural spread of tumor can occur in an antegrade or retrograde direction, the latter being more frequent.

The best imaging modality to study perineural tumor involvement is MRI, especially with T1W fat suppressed contrast enhanced sequences that demonstrate the abnormal areas of nerve enhancement. Plain T1WI sequences are also very useful because they can also show the obliteration of the normal perineural and foraminal
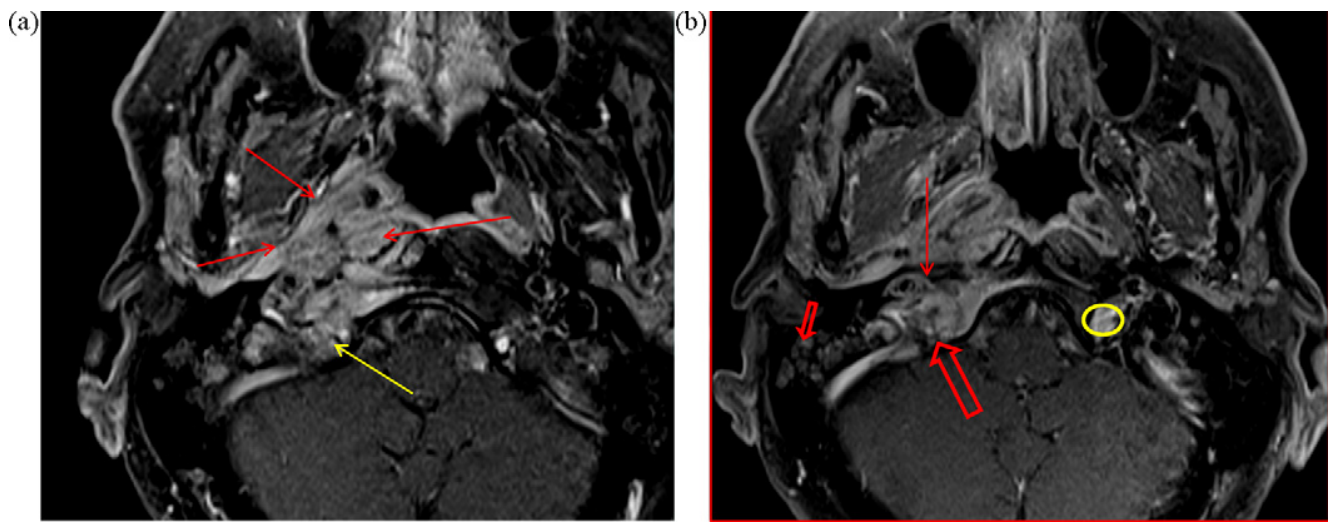

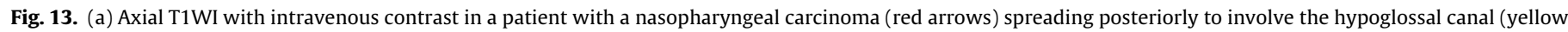

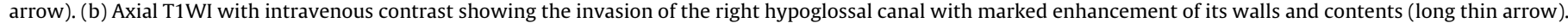
Serous otitis media is appreciated on the same side (short open arrow). The normal hypoglossal canal is shown on the left for comparison (circle).
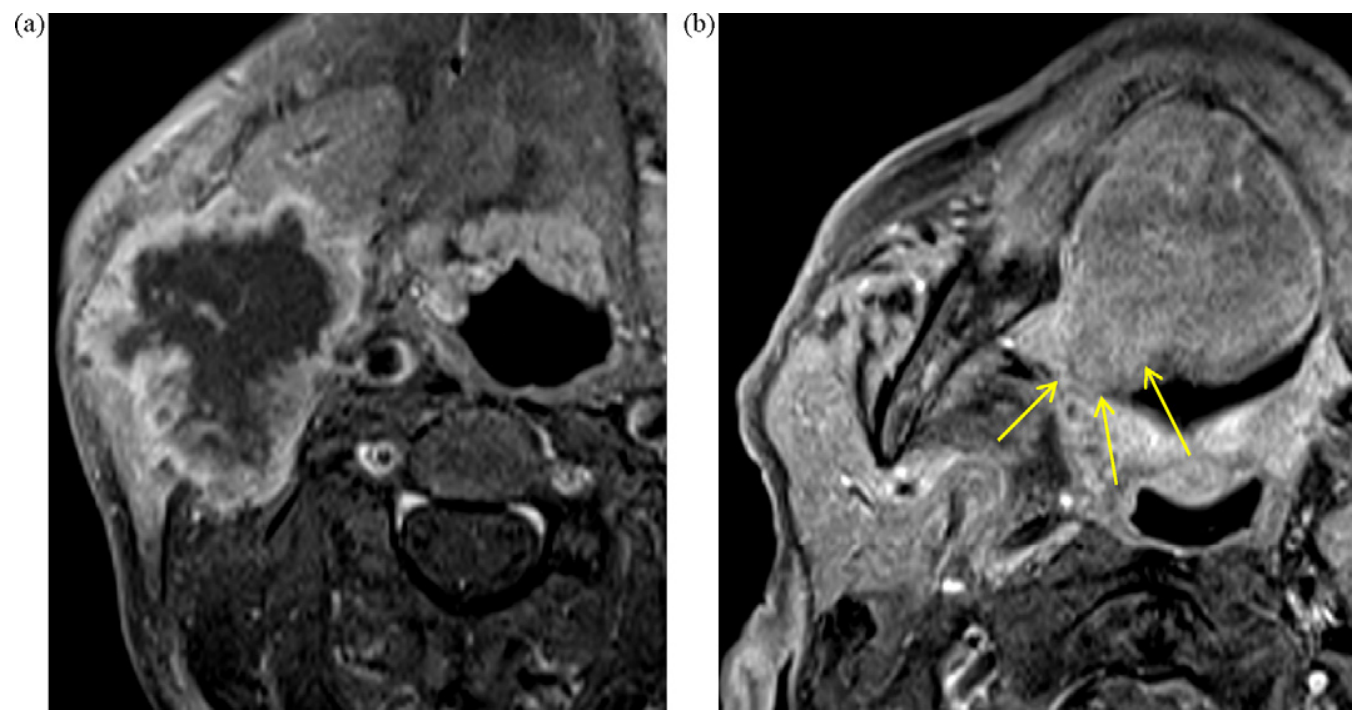

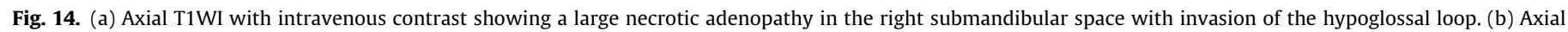
T1WI. The patient had a slight tongue dysfunction and some slices above, a subtle posterior tongue drooping (arrows) on the right could be appreciated. 


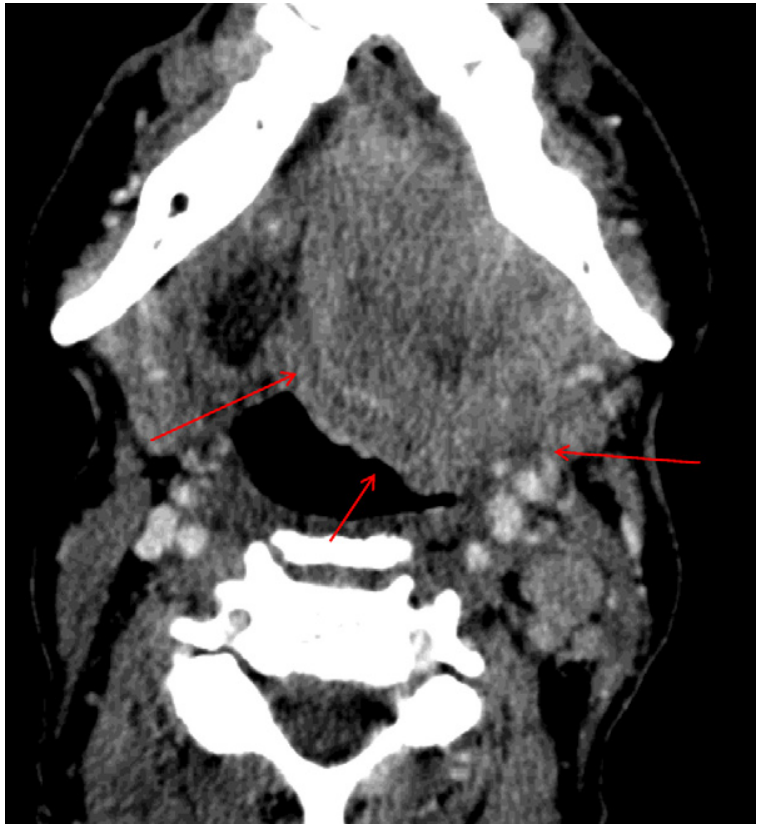

Fig. 15. Large left tongue and floor of mouth squamous cell carcinoma (arrows) with invasion of the hypoglossal nerve. The lesion crosses the midline.

fat pads, thickening of the nerve and enlargement of the hypoglossal canal.

\subsection{Extracranial segment-carotid space}

In the carotid space the hypoglossal nerve lies in intimate contact with the nearby vascular structures, namely the internal carotid artery and the internal jugular vein. Vascular pathologies, spontaneous or iatrogenic, can easily affect the nerve due to the close proximity of the vessel.

Carotid artery aneurysms or ectasia, dissection and venous thrombosis can lead to HNP by compression. Medical procedures such as endarterectomy and vascular puncture can also cause nerve dysfunction $[35,36]$.

However, as stated above, the most common causes of HNP in the carotid space are malignant tumors, both primary and secondary (Fig. 14). Squamous cell carcinoma, lymphoma, salivary gland malignancies and sarcomas are just some examples of the most common tumors.

Benign tumors, such as paragangliomas and lipomas should also be in the differential diagnosis of a carotid space lesion and as possible cause of HNP.

In the same way as iatrogenic lesions, trauma to the carotid space from stab, gunshot wounds or infection spreading from other neck spaces may all cause dysfunction of the twelfth nerve.

\subsection{Extracranial segment-anterior segment}

In this segment, where the hypoglossal nerve loops anteriorly to end in the sublingual space, the most common cause of dysfunction is nerve damage due to squamous cell carcinoma of the base or lateral regions of the tongue.

An important sign of hypoglossal nerve involvement in malignant neoplasms of the floor of mouth (Fig. 15) and tongue tumors is the obliteration of the normal fat planes adjacent to the take-off of the proximal lingual artery, a finding initially described on CT scans but also easily appreciated on MRI studies [36].

Tongue dysfunction after neck dissection for cervical adenopathy is a possibility [37], especially at the submandibular triangle where the nerve is crossed by the digastric muscle. When there is lymph node involvement of the submandibular triangle there is an increased risk of hypoglossal damage after neck dissection.

Odontogenic lesions such as abscesses or iatrogenic damage after tooth extraction are other causes to be considered in the appropriate clinical scenarios.

\section{Conclusion}

The radiological approach to patients with lesions of the hypoglossal nerve requires a good knowledge of the clinical syndromes associated with nerve damage and of the normal and radiological nerve anatomy in order to plan the most adequate study to the patient's clinical presentation.

A systematic, segmented approach seems to be the most effective way to narrow down the many diagnostic possibilities in a way that is most useful both to the clinician and the radiologist.

\section{References}

[1] Rouvière H, Delmas A. Anatomie humaine, descriptive, topographique et fonctionnelle. 13th ed. Paris: Masson; 1992

[2] Harnsberger HR, Dillon WP. Major motor atrophic patterns in the face and neck: CT evaluation. Radiology 1985;155:665-70.

[3] Smoker WRK, Harnsberger HR, Osborn AG. The hypoglossal nerve. Semin Ultrasound CT MR 1987;8:301-12.

[4] Naidich TP, Pudlowsky RM, Leeds NE, et al. Hypoglossal palsy: computed tomography demonstration of denervation hemiatrophy of the tongue associated with glomus jugulare tumor. J Comput Assist Tomogr 1978;2:630-6.

[5] Lazorthes G. Le système nerveux periphérique, le nerf grand hypoglosse. 3rd ed. Paris: Masson; 1976.

[6] Bhuller A, Sañudo JR, Choi D, Abrahams PH. Intracranial course and relations of the hypoglossal nerve. An anatomic study. Surg Radiol Anat 1998;20: 109-12.

[7] Yousry I, Moriggi B, Schmid U, et al. Detailed anatomy of the intracranial segment of the hypoglossal nerve: neurovascular relationships and landmarks on magnetic resonance imaging sequences. J Neurosurg 2002;96:1113-22.

[8] Leblanc A. The cranial nerves. Anatomy, imaging, vascularisation. 2nd ed. New York: Springer-Verlag; 1995.

[9] Kirdani MA. The normal hypoglossal canal. AJR 1967;99:700-4.

[10] Shiozawa Z, Koike G, Seguchi K, et al. Unilateral tongue atrophy due to an enlarged emissary vein in the hypoglossal canal. Surg Neurol 1996;45:477-9.

[11] Stuckey SL. Dilated venous plexus of the hypoglossal canal mimicking disease. AJNR 1999;20:157-8.

[12] Som PM, Curtin HD. Head and neck imaging. 4th ed. St Louis, MO: Mosby; 2003.

[13] Borges A, Casselman J. Imaging the cranial nerves: Part I: Methodology infectious and inflammatory, traumatic and congenital lesions. Eur Radio 2007; $17: 2112-25$.

[14] Borges A. Trigeminal neuralgia and facial nerve paralysis. Eur Radiol 2005;15:511-33.

[15] Yousry I, Camelio S, Schmid UD, et al. Visualization of cranial nerves I-XII: value of 3D CISS and T2 weighted FSE sequences. Eur Radiol 2000;10:1061-7.

[16] Keane JR. Twelfth-nerve palsy. Analysis of 100 cases. Arch Neurol 1996;53:561-6.

[17] Lin Y, Jen YM, Lin JC. Radiation-related cranial nerve palsy in patients with nasopharyngeal carcinoma. Cancer 2002;95(2):404-9.

[18] Brazis PW, Masdeu JC, Biller J. Localization in clinical neurology. 5th ed. Lippin cot Williams and Wilkins; 2006.

[19] Rontal E, Rontel M. Lesions of the hypoglossal nerve - diagnosis, treatment and rehabilitation. Laryngoscope 1982;92:927-37.

[20] Boban M, Brinar V, Habek M, Rados M. Isolated hypoglossal nerve palsy: a diagnostic challenge. Eur Neurol 2007;58:177-81.

[21] Thompson E, Smoker W. Hypoglossal nerve palsy: a segmental approach. Radiographics 1994;14:939-58.

[22] Murakami R, Nishimura R, Furusawa M, et al. CT and MR findings of denervated tongue after radical neck dissection. AJNR 1997;18:747-50.

[23] Fleckenstein JL, Watumull D, Conner KE, et al. Denervated human skeletal muscle: MR imaging evaluation. Radiology 1993;187:213-8.

[24] Naidich TP, Pudlowski RM, Leeds NE, Deck MDF. Hypoglossal palsy: computed tomography demonstration of denervation hemiatrophy of the tongue associated with glomus jugulare tumor. J Comput Assist Tomogr 1978;2:630-2.

[25] Mandrioloi J, Zini A, Cavalleri J, et al. Isolated hypoglossal nerve palsy due to amyloid cervical arthropathy in long term hemodialysis. J Neurol 2006;253:1229-31

[26] Odake G. Intracranial hypoglossal neurinoma with extracranial extension review and case report. Neurosurgery 1989;24:583-7.

[27] Ogawa T, Kitagawa Y, Ogasawara T. A multifocal neurinoma of the hypoglossal nerve with motor paralysis confirmed by electromyography. J Oral Maxillofac Surg 2001;30(2):176-8. 
[28] Passacantilli E, Lanzino G, Henn JS, Partovi S, Spetzler RF. Intracranial extradural schwannoma of the 12 th cranial nerve. Case illustration. J Neurosurg 2003;98(1):211.

[29] Rachinger J, Fellner FA, Trenkler J. Dumbbell-shaped hypoglossal schwannoma. A case report. Magn Reson Imaging 2003;21(2):155-8.

[30] Ranta A, Winter WC, Login IS. Extracranial hypoglossal schwannoma. Neurology 2003;60:E11.

[31] Zakrzewska JM. Diagnosis and differential diagnosis of trigeminal neuralgia. Clin J Pain 2002;18(1):14-21.

[32] Chandler JR, Grobman L, Quencer R, Serafini A. Osteomyelitis of the base of the skull. Laryngoscope 1986;96:245-51.

[33] Valvassori GE, Kirdani MA. The abnormal hypoglossal canal. AJR 1967;99:705-11.
[34] Dehn TCB, Taylor GW. Cranial and cervical nerve damage associated with carotid endarterectomy. BR J Surg 1983;70:365-8.

[35] Pozzo GD, Mascalchi M, Fonda C, et al. Lower cranial nerve palsy due to dissection of the internal carotid artery: CT and MR imaging. J Comput Assist Tomogr 1989;13:989-95.

[36] Dubin MG, Ebert CS, Mukherji Suresh K, Pollock HW, Amjadi D, Shockley WW. Computed tomography's ability to predict sacrifice of Hypoglossal nerve at resection. Laryngoscope 2002;112(12):2181-5.

[37] Ewing MR, Martin H. Disability following radical neck dissection; an assessment based on the postoperative evaluation of 100 patients. Cancer 1952;5:87383. 